potenciales. Este fue un estudio retrospectivo con una pequeña cantidad de casos y se llevó a cabo en una sola institución; además, no se estudiaron los subtipos de linfocitos ni otros indicadores del sistema inflamatorio, como las citocinas, la proteína C-reactiva y la sedimentación, y los antecedentes cardíacos de los pacientes no presentaban particularidades.

\section{CONCLUSIONES}

Los niveles de VPM, INL e ITL pueden ser útiles para el diagnóstico de mordedura de serpiente, el cual a veces se torna difícil para los pediatras. En este estudio no se observó una relación entre el VPM, el INL y el ITL y la gravedad de la mordedura de serpiente. Por lo tanto, se concluye que el VPM, el INL y el ITL no serían indicadores pronósticos confiables en los niños con mordedura de serpiente. Sin embargo, se requieren investigaciones futuras con mayor cantidad de pacientes para establecer si existe una relación entre estos biomarcadores y la gravedad de la forma clínica en los niños con mordedura de serpiente.

\section{REFERENCIAS}

1. Aktar F, Aktar S, Yolbas I, et al. Evaluation of risk factors and follow-up criteria for severity of snakebite in children. Iran J Pediatr 2016;26(4):e5212.

2. Chippaux JP. Epidemiology of snakebites in Europe: a systematic review of the literature. Toxicon 2012;59(1): 86-99.

3. Gold BS, Dart RC, Barish RA. Bites of venomous snakes. N Engl J Med 2002;347(5):347-56.

4. Santhosh MS, Sundaram MS, Sunitha K, et al. Viper venom- induced oxidative stress and activation of inflammatory cytokines: a therapeutic approach for over looked issues of snakebite management. Inflamm Res 2013;62(7):721-31.

5. Sunitha K, Hemshekhar M, Thushara RM, et al. Inflammation and oxidative stress in viper bite: an insight within and beyond. Toxicon 2015;98:89-97.

6. Mannaioni PF, Di Bello MG, Masini E. Platelets and inflammation: role of platelet-derived growth factor, adhesion molecules and histamine. Inflamm Res 1997;4 6(1):4-18.

7. Otero-Patiño R. Epidemiological, clinical and therapeutic aspects of Bothrops asper bites. Toxicon 2009;54(7): 9981011.

8. Tekin R, Sula B, Cakır G, et al. Comparison of snakebite cases in children and adults. Eur Rev Med Pharmacol Sci 2015;19(14):2711-6.

9. Kemal Y, Yucel I, Ekiz K, et al. Elevated serum neutrophile to lymphocyte and platelet to lymphocyte ratios could be useful in lung cancer diagnosis. Asian Pac J Cancer Prev 2014;15(6):2651-4.

10. Bhat T, Teli S, Rijal J, et al. Neutrophil to lymphocyte ratio and cardiovascular diseases: a review. Expert Rev Cardiovasc Ther 2013;11(1):55-9.

11. Ulu S, Ulu MS, Bucak A, et al. Neutrophil-to-lymphocyte ratio as a new, quick, and reliable indicator for predicting diagnosis and prognosis of idiopathic sudden sensorineural hearing loss. Otol Neurotol 2013;34(8):1400-4.

12. Dinarello CA. Biologic basis for interleukin-1 in disease. Blood 1996;87(6):2095-147.

13. McCleary RJ, Kini RM. Snake bites and hemostasis/ thrombosis. Thromb Res 2013;132(6):642-6.

14. Feng JF, Huang Y, Chen QX. Preoperative platelet lymphocyte ratio (PLR) is superior to neutrophil lymphocyte ratio (NLR) as a predictive factor in patients with esophageal squamous cell carcinoma. World J Surg Oncol 2014;12:58.

15. Acmaz G, Aksoy H, Unal D, et al. Are neutrophil/ lymphocyte and platelet/lymphocyte ratios associated with endometrial precancerous and cancerous lesions in patients with abnormal uterine bleeding? Asian Pac J Cancer Prev 2014;15(4):1689-92.

\title{
Participación de la mujer en la autoría de revistas pediátricas latinoamericanas
}

\section{Female authorship in Latin American pediatric journals}

\author{
Dra. Paula Otero ${ }^{a, b}$, Cecilia Marcos ${ }^{b}$ y Dr. Fernando Ferrero ${ }^{b, c}$
}

\author{
a. Hospital Italiano de Buenos Aires. \\ b. Archivos Argentinos de Pediatría. \\ c. Hospital General de Niños Pedro de Elizalde.
}

Correspondencia: Dr. Fernando Ferrero,fferrero@intramed.net.

Financiamiento: Ninguno.

Conflicto de intereses: Ninguno que declarar.

Recibido: $19-1-2017$

Aceptado: 12-6-2017

\section{RESUMEN}

Introducción. La participación de la mujer en las ciencias se incrementa día a día. Se estimó la participación de la mujer en las 3 publicaciones pediátricas latinoamericanas indexadas en PubMed.

Métodos. Se identificaron todos los artículos de Archivos Argentinos de Pediatría, Jornal de Pediatria y Revista Chilena de Pediatría correspondientes al año 2015 y se determinó el primer y último autor, y el número total de autores por sexo.

Resultados. Se identificaron 329 artículos. De los 1432 autores, $59,9 \%$ eran mujeres. El $54,4 \%$ de los primeros autores y el $48 \%$ de los últimos autores eran mujeres. No hubo diferencia significativa en la proporción de mujeres autoras entre las 
revistas. Archivos Argentinos de Pediatría tuvo significativamente menos mujeres como primer y último autor.

Conclusión. La proporción de mujeres autoras en tres revistas pediátricas latinoamericanas alcanzó el 59,9\%. La participación como primer o último autor fue significativamente menor en Archivos Argentinos de Pediatría.

Palabras clave: autoría, informe de investigación, mujeres, publicaciones periódicas.

http:/ / dx.doi.org/10.5546/ aap.2017.580

Texto completo en inglés:

http:/ / dx.doi.org/10.5546/ aap.2017.eng.580

Cómo citar: Otero P, Marcos C, Ferrero F. Participación de la mujer en la autoría de revistas pediátricas latinoamericanas. Arch Argent Pediatr 2017;115(6):580-583.

\section{INTRODUCCIÓN}

La participación de la mujer en la medicina ha crecido en forma notable durante el siglo XX. Como menciona Filardo, ${ }^{1}$ la proporción de mujeres médicas llega a 33\% en Estados Unidos de América (EE. UU.), 47\% en Reino Unido (RU) y más de 50\% en Europa del Este. En Argentina, la proporción de mujeres médicas alcanza el 50\% (60\% en menores de 40 años) ${ }^{2}$ en Brasil, $40 \%$ (53\% en menores de 30 años), ${ }^{3} \mathrm{y}$, en Chile, $33 \%$ (46\% en menores de 30 años). ${ }^{4}$ En pediatría, la participación de la mujer es aún más evidente, según la proporción de miembros femeninos de las sociedades de pediatría de Argentina (9044/12772; 70,8\%), Brasil (16372/22158; 74\%) y Chile $(1393 / 2880 ; 48,3 \%$ )(datos proporcionados por las respectivas sociedades en abril de 2017).

A pesar de ese equilibrio cuantitativo al que parece estar llegándose, aún existirían diferencias en el acceso a ciertos ámbitos. Por ejemplo, en Argentina, 73,1\% de los alumnos de Medicina de la Universidad de Buenos Aires son mujeres, ${ }^{5}$ pero solo el $53,7 \%$ de los docentes lo son. ${ }^{6}$

La actividad académica, especialmente la que desemboca en la publicación científica, es uno de los sectores en los que pueden presentarse diferencias atribuibles al sexo y, aunque la situación ha mejorado considerablemente, todavía las mujeres podrían estar sub-representadas, sobre todo, en las posiciones de mayor jerarquía. ${ }^{7}$

Hace 10 años, en pediatría y en nuestra región, la participación de la mujer en la publicación científica era aún limitada, pero mostraba un incremento en los últimos años. ${ }^{8}$

Nuestro objetivo fue estimar la participación de las mujeres como autoras de los trabajos publicados en tres revistas pediátricas latinoamericanas indexadas en PubMed, en el año 2015.

\section{MATERIAL Y MÉTODOS}

Se identificaron todos los artículos publicados en tres revistas de pediatría latinoamericanas indexadas en PubMed, en 2015: Archivos Argentinos de Pediatría (Argentina), Jornal de Pediatria (Brasil) y Revista Chilena de Pediatría (Chile). Este procedimiento se llevó a cabo mediante la búsqueda en la base de referencias bibliográficas Literatura Latinoamericana y del Caribe en Ciencias de la Salud (LILACS:http: / / lilacs.bvsalud.org/es/), complementada con la búsqueda en el sitio web de cada publicación cuando el dato no estaba disponible en LILACS. ${ }^{8}$

Las referencias comprendían trabajos originales, reportes de casos, revisiones y editoriales. No se incluyeron comentarios de otras publicaciones.

En los artículos seleccionados, se determinó el sexo del primer y último autor, y el número total de autores por sexo para cada una de las publicaciones y se expresó en porcentaje (calculando el IC95\% en los resultados principales).

Para cada variable (primer autor, último autor, número total de autores), se evaluó si existía asociación entre el sexo y las revistas seleccionadas por medio de la prueba de $\chi^{2}$. Se aceptó como valor de significación $p<0,05$.

Consideraciones éticas: Todos los datos utilizados en este trabajo eran de dominio público y se encontraban disponibles en internet.

\section{RESULTADOS}

Se incluyeron en el análisis un total de 329 artículos, que correspondían a 1432 autores, en los que se pudo identificar el sexo (en 5 casos, no se pudo identificar el sexo a partir del nombre o grado académico) (Tabla 1).

La proporción de mujeres autoras $(59,9 \%$; IC95\%: 57,3-62,4) no mostró diferencias significativas entre las 3 publicaciones. El 54,4\% de los primeros autores y el $48 \%$ de los últimos autores eran mujeres.

La proporción de mujeres primeras y últimas autoras fue significativamente menor en Archivos Argentinos de Pediatría que en las otras dos publicaciones (Tabla 1).

\section{DISCUSIÓN}

La proporción de mujeres autoras en revistas pediátricas latinoamericanas es cercana al $60 \%$. Ese valor se ha incrementado en forma sostenida en los últimos 30 años: $38 \%$ en 1985, $42 \%$ en 1995 y 48\% en 2005 (8). Algo similar ocurrió en 
relación con su participación como primeras autoras, ya que había sido reportado $27 \%$ en 1985 , $34 \%$ en $1995,42 \%$ en $2005^{8} \mathrm{y}$, en este trabajo, se encontró $54 \%$ en 2015 . Esta tendencia también ha sido reportada en los últimos 20 años para las principales revistas de medicina interna. ${ }^{1}$

Es posible que una mayor participación en equipos de investigación y acceso a subsidios de investigación pueda tener alguna influencia en esto. Por ejemplo, la proporción de investigadoras mujeres se ha incrementado hasta alcanzar $53 \%$ en Argentina, 36\% en Brasil y 32\% en Chile. ${ }^{9}$ En Argentina, este incremento ha sido, fundamentalmente, a expensas de becarios ${ }^{10} \mathrm{y}$ de los profesionales que se incorporan a la Carrera de Investigador.

En relación con su participación como últimas autoras, si bien, luego de mantenerse relativamente estable por cerca de 20 años $(1985=35,8 \%, 1995=29,4 \%, 2005=38,1 \%){ }^{8}$ se ha registrado un notable incremento $(48 \%$ en 2015), aún no alcanza la magnitud de los otros indicadores. Es posible que la participación de las mujeres en las actividades de investigación se haya incrementado, pero que este incremento todavía no se refleje en los puestos de mayor jerarquía. En la agencia argentina de investigación científica (Consejo Nacional de Investigaciones Científicas y Técnicas, CONICET), en el año 2013, en la categoría inicial (investigador asistente), 57\% eran mujeres, pero solo 37\% de ellas alcanzaban la categoría más alta (investigador superior). ${ }^{11}$

Recientemente, Macaluso y col., analizando 85000 artículos publicados en revistas pertenecientes a la Public Library of Science (PLOS), encontraron que la participación de las mujeres en las publicaciones científicas se asociaba significativamente al rol de "desarrollar el experimento". Esto ocurría con independencia de sus años de vida académica, cuando era de esperar que a mayor "edad académica" se participara en otros roles identificados como de mayor jerarquía (diseño del estudio, redacción del manuscrito). ${ }^{12}$

Además, existen factores tan variados como la "capacidad de controlar la cantidad de trabajo asignado" o la "posibilidad de sentirse exhausto en el trabajo en desarrollo", en los que se observan diferencias por sexo y que podrían influir en la producción científica, ${ }^{13}$ lo que ayudaa explicar, al menos de modo parcial, las diferencias observadas en autoría.

Finalmente, no es fácil explicar la diferencia encontrada entre las tres publicaciones evaluadas. Son las principales revistas pediátricas de sus respectivos países y todas se encuentran indexadas en PubMed (Jornal de Pediatria: 2003; Archivos Argentinos de Pediatría: 2008; y Revista Chilena de Pediatría: 2014).

Desde hace 3 años, Archivos Argentinos de Pediatría publica sus principales contenidos en español e inglés, y recibe una importante participación extranjera, particularmente de Iberoamérica; esto podría haber sumado el sesgo de género de otros países. También se debería tener en cuenta el posible impacto que la composición de los respectivos cuerpos editoriales y de revisores podría tener. ${ }^{14}$

A pesar de las limitaciones de este tipo de inferencia, la posibilidad de comparar los resultados con los de otra investigación desarrollada con la misma metodología ${ }^{8}$ permite estimar una tendencia aún en ascenso y especular que la participación de la mujer en estas publicaciones no ha alcanzado todavía su punto más alto.

TABLA 1. Distribución de los autores según género y por publicación, en 3 revistas pediátricas latinoamericanas indexadas en PubMed

\begin{tabular}{|c|c|c|c|c|c|c|c|c|}
\hline & \multirow[t]{2}{*}{ Artículos } & \multirow{2}{*}{ Autores } & \multicolumn{2}{|c|}{ Mujer autora* } & \multicolumn{2}{|c|}{ Mujer primera autora** } & \multicolumn{2}{|c|}{ Mujer última autora ${ }^{* * *}$} \\
\hline & & & $\mathbf{n}$ & $(\%)$ & $\mathbf{n}$ & $(\%)$ & $\mathbf{n}$ & $(\%)$ \\
\hline Archivos Argentinos de Pediatría & 156 & 771 & 447 & $(57,9)$ & 71 & $(45,5)$ & 66 & $(42,3)$ \\
\hline Jornal de Pediatria ${ }^{\mathrm{b}}$ & 103 & 385 & 241 & $(62,5)$ & 63 & $(61,1)$ & 57 & $(55,3)$ \\
\hline Revista Chilena de Pediatría & 70 & 276 & 170 & $(61,5)$ & 45 & $(64,2)$ & 35 & (50) \\
\hline Total & 329 & 1432 & 858 & $(59,9)$ & 179 & $(54,4)$ & 158 & (48) \\
\hline
\end{tabular}

${ }^{*} \mathrm{p}>0,1\left({ }^{\mathrm{a}}\right.$ vs. ${ }^{\mathrm{b}},{ }^{\mathrm{b}}$ vs. ${ }^{\mathrm{c}} \mathrm{y}^{\mathrm{a}}$ vs. $\left.{ }^{\mathrm{c}}\right)$.

${ }^{* *} \mathrm{p}<0,05\left({ }^{\mathrm{a}}\right.$ vs. $\left.{ }^{\mathrm{b}, \mathrm{c}}\right)$.

${ }^{* * *} \mathrm{p}<0,01\left({ }^{\mathrm{a}}\right.$ vs. $\left.{ }^{\mathrm{b}, \mathrm{c}}\right)$. 
Cabe destacar que la desigualdad de género que se observó no solo abarca al mundo académico, ya que se muestra en otros ámbitos laborales y aún impacta en el desempleo, que afecta mayormente a las mujeres, en especial a las más jóvenes. ${ }^{15}$

\section{CONCLUSIÓN}

La presencia de mujeres autoras en artículos publicados en revistas de América Latina se ha incrementado en forma sostenida en las últimas décadas. En este estudio, la proporción de mujeres autoras en tres revistas pediátricas latinoamericanas alcanzó el 59,9\%. La participación como primeras o últimas autoras fue significativamente menor en Archivos Argentinos de Pediatría.

\section{Agradecimiento}

Los autores agradecen a los presidentes de la Sociedad Argentina de Pediatría, Sociedade Brasileira de Pediatria y Sociedad Chilena de Pediatría, quienes gentilmente compartieron los datos sobre sus respectivas sociedades.

\section{REFERENCIAS}

1. FilardoG, da GracaB, Sass DM, et al. Trends and comparison of female first authorship in high impact medical journals: observational study (1994-2014). BMJ 2016;352:i847.

2. Argentina. Ministerio de Salud. Observatorio Nacional de Recursos Humanos en Salud. Los recursos humanos de salud en la Argentina. 2015. [Acceso: 23de diciembre de 2016]. Disponible en: http://www.msal.gob.ar/ observatorio/images/stories / documentos_fuerza_ trabajo/RHuS_ARG_2015.pdf.

3. Scheffer M, Cassenote A. A feminização da medicina no Brasil. Ver Bioet 2013;21(2):268-77.
4. Instituto Nacional de Estadística, Chile. Compendio Estadístico. 1.6 Estadísticas de Salud 2010. [Acceso: 3 de abril de 2017]. Disponible en: $h t t p: / / w w w . i n e . c l / d o c s / d e f a u l t-$ source/publicaciones/2010/compendio_2010.rar?sforsn=5.

5. Universidad de Buenos Aires. Censo de estudiantes 2011. [Acceso: 16 de diciembre de 2016]. Disponible en: http:/ / www.uba.ar/institucional / censos / Estudiantes2011/ estudiantes2011.pdf.

6. Universidad de Buenos Aires. Censo de docentes 2011. [Acceso: 16 de diciembre de 2016].Disponible en:www. uba.ar/institucional/censos/Docentes2011/docentes2011final.pdf.

7. West JD, Jacquet J, King MM, et al. The Role of Gender in Scholarly Authorship. PLoSOne 2013;8(7):e66212.

8. FerreroF, OteroP.Participación dela mujeren publicaciones periódicas científicas: tendencias en la pediatría latinoamericana. Arch Argent Pediatr 2007;105(3):244-7.

9. UNESCO InstituteforStatistics. Mujeres en ciencia. Investigadoras por país. [Acceso: 4 de abril de 2017]. Disponibleen:http:/ / www.uis.unesco.org/_LAYOUTS / UNESCO/women-in-science/index.html? $\mathrm{t}=\overline{1} 49124378263$ 7\# overview!lang=es\&region $=40520$.

10. Red de Indicadores de Ciencia y Tecnología Iberoamericana e Interamericana. Recursos humanos. Personal por género. [Acceso: 12 de diciembre de 2016]. Disponible en: http: / / db.ricyt.org/ query / AR,BO,CL,CO,CR,CU,EC,ES ,GT,HN,NI,PA,PR,PT,PY,SV,TT,UY,VE/1990\%2C2014 / PERSOPFGENPER.

11. Franchi A, Kochen S, Maffía D, et al. Evolución de la situación de las Mujeres en el sector de Ciencia y Tecnología en Argentina (1998-2013). X Congreso Iberoamericano de Ciencia, Tecnología y Género. 28 a 30 de octubre de 2014. - Asunción, Paraguay; 2014.

12. Macaluso B, Larivière $V$, Sugimoto $T$, et al. Is Science Built on the Shoulders of Women? AStudy of GenderDifferences in Contributorship. Acad Med 2016;91(8):1136-42.

13. Fridner A, Norell A, Åkesson G, et al. Possible reasons why female physicians publish fewer scientific articles than male physicians - a cross-sectional study. BMC Med Educ 2015;15:67.

14. DelBocaFK. Addressing sexand genderinequitiesinscientific research and publishing. Addiction 2016;111(8):1323-5.

15. Organización Internacional del Trabajo. Las mujeres en el trabajo. Tendencias 2016. Resumen ejecutivo. Ginebra, 2016. [Acceso: 4 de abril 2017]. Disponible en:http: / www. unesco.org/library/PDF/wcms_457094.pdf

\title{
Estrategias para el cumplimiento del programa de formación en tres médicos residentes de Pediatría con alergia al látex
}

\author{
Strategies for compliance with the internship program among three pediatric \\ interns with latex allergy
}

Dra. Stefanía Barbariol ${ }^{a}$ Dr. Alfredo Eymann ${ }^{b, c}$, Dr. Julián Llera ${ }^{c}$ y Dr. Claudio A. Parisi ${ }^{a}$

a. Sección de Alergia del Servicio de Clínica Pediátrica del Hospital Italiano de Buenos Aires, Buenos Aires, Argentina.

b. Departamento de Posgrado del Instituto Universitario del Hospital Italiano de Buenos Aires, Buenos Aires, Argentina.

c. Servicio de Clínica Pediátrica del Hospital Italiano de Buenos Aires, Buenos Aires, Argentina.

\author{
Correspondencia: \\ Dr. Claudio A. Parisi: claudio.parisi@hospitalitaliano.org.ar \\ Financiamiento: Ninguno. \\ Conflicto de intereses: Ninguno que declarar.
}

Recibido: 28-2-2017

Aceptado: 28-6-2017 